contradiction, although they refer to concepts that can be given a precise connotation within a theory. Schlegel seems to accept this in principle (page 151) but fails to fasten the reader's attention to its importance.

This review has been almost entirely critical: I hope Schlegel would regard it as a response to his para-Gödelian sentence, "I would have known that I had failed if I had come to a final answer". My comments are intended to supply a small contribution towards the strengthening of what appear to me to be a few weaknesses in the philosophical foundations in a book that has made mo think and think hard. I hope that it will engage the thoughts of a wide circle of readers. It is admirably produced: only two trivial typographical slips caught my eye.

WILliam P. D. WrghtMaN

\section{ODDMENTS OF OPTIMIZATION}

\section{Topics in Optimization}

Edited by George Leitmann. (A Series of Monographs and Textbooks on Mathematics in Science and Engineering, Vol. 31.) Pp. xv +469. (New York: Academic Press' Inc.; London: Academic Press, Inc. (London), Ltd., 1967.) $\$ 18.00$.

ThIs book is a collection of ten papers dealing with various aspects of optimal control theory. The stated purpose of the collection is to record in permanent format worthwhile contributions which are too long for inclusion in technical journals. Some material in the longest contribution, "On the Geometry of Optimal Processes", by Blaquiere and Leitman, however, has already appeared in book form, while several other contributions are short enough to appear in journals. Indeed, as in other similar collections, it is unlikely that more than one or two of the contributions will be of particular interest to a reader. This book is, however, distinguished by a most interesting and important contribution by Halkin, "Mathematical Foundations of System Optimisation". In this chapter Halkin outlines a geometric proof of the maximum principle, which he developed several years ago, and whose exposition he has refined in successive papers. By means of a new theorem he is now able to develop the proof "without introducing measurable control functions which are mathematical artifacts, completely out of place in control theory". This simplification makes the proof understandable by a relatively wide aud ence, and will be much appreciated. This chapter is, without doubt, the most important in the book. Another useful and interesting contribution is the chapter on singular extremals by Kelley, Kopp and Moyer. The maximum principle does not provide an adequate test for optimality on singular subarcs; this results in the failure of normal gradient methods of optimization. Singular extremals occur in problems of practical interest, and so the development of suitable numerical procedures is an important task. This is not done in this contribution, but a useful derivation of the conditions of minimality of singular ares over short intervals of time is presented. The chapter by Blaquiere and Leitman presents (in 107 pages) a geometric approach to optimal processes which is at first sight very appealing, but which leads to considerable complexity. Halkin's proof of the maximum principle is undoubtedly preferable. The remaining contributions are either actually or potentially short enough for publication in journals, and deal with: bang-bang principle for non-linear systems; the relationship between classical calculus of variations, functional analysis and optimal control theory; inequalities and discontinuities in variational problems; determ. ination of optimal control of a boosting vehicle; optimal problems in the theory of magnetohydrodynamics; numerical methods for the determination of minimum time control.

D. Q. MAYNE

\section{INFRARED SPECTRA}

\section{Interpreted Infrared Spectra}

Vol. 3. Including a Cumulative Index. By Herman A. Szymanski. Pp. ix +275 . (New York: Plenum Press Data Division, 1967.) $\$ 12.50$.

THE third volume of Szymanski's Interpreted Infrared Spectra is devoted to phenols, ethers and peroxides. For each class, spectra with vibrational assignments are presented for typical compounds. This is followed by a short discussion of correlations and a table of appropriate group frequencies. Then the spectra of some fifty or sixty compounds in each class are reproduced, attention being drawn to the various group frequencies and any special points in each case. The aim is to provide instruction in spectral interpretation by showing how the significant bands may be identified and recognized in the spectra of related compounds, even where interfering bands may be encountered in some instances.

A short literature survey on phenols covers hydrogen bonding, and the effects of ortho- and para-substituents. Ethers are dealt with at some length in two separate sections, non-cyclic and cyclic ethers. Group frequencies for peroxides and hydroperoxides are discussed in relation. to ethers and alcohols respectively. Some tentative $-\mathrm{O}-\mathrm{O}-$ stretching frequencies are assigned but this band has only a limited diagnostic value.

The reasons underlying the choice of spectra are not always clear. Thus for the first member of the ethers, dimethyl ether assignments reported in 1945 and 1957 are given but more recent work is not included. Consequently, the book has limited value to those interested in detailed assignments. It will be of most value to those who wish to make use of group frequencies and know the class of compound they are working with. Interestingly enough, the author confesses to the view that where it is a question of identifying an unknown from an infrared spectrum he is convinced the only method is an exact matching of each band using a computer sort.

A cumulative index covering not only phenols, ethers and peroxides, but also alcohols and alkynes, is provided. The book is splendidly produced, tables well set out, and spectra clearly reproduced.

D. J. Millen

\section{LINEAR ALGEBRA}

\section{Linear Algebra}

By Walter Nef. Translated from the German by J. C. Ault. (European Mathematics Series.) Pp. $x+305$. London : McGraw-Hill Publishing Company, Ltd., (1967.) 80s.

There are many books on linear algebra. What is different about this one? The special feature is the blending of pure and applied aspects of the subject. This book is not a practical primer on the matrix calculus, such as might be useful to those engaged in computation; and it is not devoted exclusively to the theory of vector spaces. and their linear mappings. Instead it lays the foundation for the theory of linear algebra and then proceeds to an introduction to linear programming and the theory of games. As a result, the book will be of special value to those students who will be concerned with applications. but who wish to understand the fundamental theory at the back of what would otherwise be little more than a set of rules and recipes.

The book opons with a short chapter on sets and mappings and then proceeds to the initial treatment of vector spaces, including the theory of cosets (linear manifolds) and quotient spaces. The next chapter is concerned with bases of a vector space, especially a space of finite dimension. It includes a discussion of the exchange method of numerical calculation. The following chapter, on determinants, starts with a treatment of permutations 